\title{
Minimizando erros na administração de drogas intravítreas
}

\author{
Minimizing errors in intra vitreal drug injection
}

\author{
Yeh Shu I \\ Bruno Castelo Branco ${ }^{2}$ \\ Érika Sayuri Yasaki ${ }^{3}$ \\ Paulo Schor $^{4}$ \\ Denise de Freitas 5 \\ Rubens Belfort $\mathbf{J r}^{6}$
}

\begin{tabular}{|c|}
\hline RESUMO \\
\hline Objetivo: Avaliar possíveis erros na administração de drogas intravítreas \\
para o tratamento da endoftalmite e propor técnica que seja reprodutível \\
e acessível. Métodos: Avaliação de técnicas utilizadas e aferição dos \\
volumes retidos nas agulhas utilizando balança analítica. Resultados: A \\
média e o desvio padrão dos volumes retidos nas agulhas de $26,22(25 \mathrm{x}$ \\
$0,7 \mathrm{~mm}$ e $30 \times 0,7 \mathrm{~mm})$ e $18 \mathrm{G}$ (gauge) foram $0,051 \pm 0,006,0,056 \pm 0,005$, \\
$0,055 \pm 0,004$ e $0,075 \pm 0,004$, respectivamente, para a marca Ryncos ${ }^{\circledast} \mathrm{e}$ \\
$0,050 \pm 0,003,0,056 \pm 0,002,0,063 \pm 0,002 \mathrm{e} 0,084 \pm 0,004$, respectivamente, \\
para a marca Becton-Dickinson ${ }^{\circledast}$. Houve diferença estatisticamente \\
significante entre os volumes retidos das duas marcas para as agulhas de \\
$26,22(30 \times 0,7$ mm) $18 \mathrm{G}$ comp $=0,01, \mathrm{p}<0,01$ ep $<0,01$ respectivamente. \\
Não foi encontrada diferença estatisticamente significante apenas para as \\
agulhas de $25 \times 0,7 \mathrm{~mm}(22 \mathrm{G})$ comp $=0,83$. Conclusão: A maioria das agulhas \\
utilizadas na injeção intravítrea comporta um volume residual no espaço \\
morto que deve ser valorizado, minimizando assim erros na injeção de \\
drogas intravítreas no tratamento da endoftalmite.
\end{tabular}

Descritores: Endoftalmite/quimioterapia; Corpo vítreo/efeitos de drogas; Antibióticos/ administração \& dosagem; Injeções/métodos; Erros de medicação

Trabalho realizado nos Setores de Doenças Externas Oculares e Córnea e de Bioengenharia Ocular do Departamento de Oftalmologia da Universidade Federal de São Paulo - UNIFESP.

${ }^{1}$ Residente de Terceiro Ano do Departamento de Oftalmologia da Universidade Federal de São Paulo UNIFESP.

${ }^{2}$ Diretor Clínico do Instituto Paulista de Estudos e Pesquisas em Oftalmologia - IPEPO, Doutor pela Universidade Federal de São Paulo - UNIFESP e PósDoutorado pela Universidade Federal de São Paulo UNIFESP.

${ }^{3}$ Estagiária do Setor de Retina do Departamento de Oftalmologia da Universidade Federal de São Paulo UNIFESP.

${ }^{4}$ Professor Afiliado, Diretor do Setor de Bioengenharia do Departamento de Oftalmologia da Universidade Federal de São Paulo - UNIFESP.

${ }^{5}$ Livre-Docente do Departamento de Oftalmologia da Universidade Federal de São Paulo - UNIFESP.

${ }^{6}$ Professor Titular do Departamento de Oftalmologia da Universidade Federal de São Paulo - UNIFESP.

Endereço para Correspondência: R. Botucatu, 822 Vila Clementino - São Paulo (SP) CEP 04023-062 E-mail:shuiyeh@ig.com.br; bcbranco@uol.com.br

Recebido para publicação em 18.10.2001 Aceito para publicação em 14.08.2002

Nota Editorial: Pela análise deste trabalho e por sua anuência na divulgação desta nota, agradecemos ao Dr. Walter Yukihiko Takahashi. $\frac{\text { INTRODUÇÃO }}{\text { A injeção intravítrea de antibióticos é um dos principais recursos tera- }}$ pêuticos utilizados no tratamento da endoftalmite, sendo, atualmente, a opção de escolha utilizada nos casos de endoftalmite com visão melhor do que percepção de movimentos de mão ${ }^{(1-3)}$. Comparando-se a concentração intra-ocular obtida através da injeção intravítrea do antibiótico com qualquer outra modalidade de administração, encontra-se maior sucesso terapêutico com a primeira. Outras vias de administração como a tópica ou a subconjuntival, não atingem níveis terapêuticos satisfatórios no vítreo ${ }^{(1-5)}$.

Vários estudos têm demonstrado reações de toxicidade retiniana induzidas pelo uso da vancomicina, ceftazidima ou aminoglicosídeos intraví$\operatorname{treos}^{(3,6-10)}$. Este alerta torna-se ainda mais importante quando se tem conhecimento do aumento na reação tóxica após injeções repetidas ${ }^{(1,3,9)}$ ou pelo preparo inadequado das soluções ${ }^{(3,7,11)}$. Este preparo inadequado pode ser devido ao fato da existência de um compartimento de reserva dentro da seringa e da agulha.

O compartimento de reserva é o espaço ocupado pelo lume da agulha e eixo plástico, e o lume do bico da seringa; estas variam de acordo com o tamanho e fabricante da agulha e da seringa. Este compartimento de reserva não tem implicação na maioria das injeções em que envolve apenas a aspiração da droga e a injeção da mesma, pois o volume extra aspirado para a seringa, correspondente ao compartimento de reserva, permanece dentro 
do conjunto agulha e seringa mesmo após a injeção. O compartimento de reserva passa a ter significado quando duas drogas são misturadas na mesma seringa, particularmente se a dose está concentrada num pequeno volume de líquido ${ }^{(12)}$. Neste particular, a injeção de antibióticos intravítreo pode ser comprometida, pois, em ambos os casos, os volumes envolvidos são extremamente pequenos.

A avaliação da toxicidade e eficácia dos antibióticos de administração intravítrea pode ser errônea se não houver uma detalhada descrição do método de injeção, inclusive a descrição dos cuidados na troca de agulhas e seringas durante a aplicação $^{(5,8-9,11,13-15)}$.

Posto isto, torna-se necessária a padronização de uma técnica de injeção que seja confiável, reprodutível e acessível a todos os oftalmologistas, para que a administração de drogas intravítreas não sofram variações que possam acarretar uma quantidade inadequada do medicamento.

O objetivo desse estudo foi determinar o volume retido nas agulhas utilizadas na injeção de antibióticos intravítreos e propor uma técnica reprodutível e acessível para esta via de administração de drogas.

\section{MÉTODOS}

Para a determinação do volume retido na agulha foi realizado pesagem do material utilizado na injeção de drogas intravítreas com uma balança analítica LIBROR AEG-45SM SHIMADZU ${ }^{\circledR}$ (capacidade 45 gramas e precisão de $10^{-5}$ gramas) dos seguintes itens:

1. Agulhas $18,22(30 \times 0,7 \mathrm{~mm}$ e $25 \times 0,7 \mathrm{~mm})$ e $26 \mathrm{G}$.

2. Seringa de 1 mililitro preenchida com água destilada com o êmbolo no marcador de $0,5 \mathrm{ml}$.

3. Seringa de $1 \mathrm{ml}$ acoplada a cada uma das agulhas e preenchida com água destilada com o êmbolo no marcador de $0,5 \mathrm{ml}$ (tomando-se o cuidado de expelir toda a bolha de ar).

Todas as medidas foram realizadas utilizando sempre a mesma seringa e com o mesmo observador, com o objetivo de eliminar a seringa e o observador como fator de variabilidade do peso.

Cinqüenta amostras de cada tipo de agulha de duas marcas diferentes (Ryncos ${ }^{\circledR}$ e Becton-Dickinson ${ }^{\circledR}$ ) foram analisadas, num total de 400 agulhas. O tamanho da amostra das agulhas foi calculado para um erro amostral de 0,03. Duas marcas de agulha, escolhidas aleatoriamente, foram testadas com o objetivo de avaliar a existência de variação do volume retido entre as diferentes marcas.

Foram feitas 10 medidas do peso de uma seringa de $1 \mathrm{ml}$ preenchida com água destilada e com o êmbolo até a marca de $0,5 \mathrm{ml}$, obtendo sua média e desvio padrão. Com base nesse valor foi feito o cálculo do volume retido de cada agulha subtraindo do peso do conjunto agulha e seringa preenchidas com água destilada, o peso da agulha vazia e o peso da seringa apenas preenchida com água destilada. Os valores foram convertidos para mililitros, considerando a densidade da água como $1 \mathrm{~g} / \mathrm{ml}$.

Foram determinadas as médias e os desvios padrão dos volumes retidos de cada tipo de agulha. A avaliação da diferença dos volumes retidos das duas marcas para cada calibre de agulha foi determinada pelo teste t-student bicaudal para amostras independentes e determinado o nível de significância de 0,05 .

\section{RESULTADOS}

No grupo das agulhas da marca Becton-Dickinson ${ }^{\circledR}$, o volume residual obtido para as agulhas $18,22(30 \mathrm{x} 0,7 \mathrm{~mm}$ e $25 \mathrm{x}$ $0,7 \mathrm{~mm})$ e $26 \mathrm{G}$ foram respectivamente $0,084 \pm 0,004 \mathrm{ml}, 0,063 \pm$ $0,002 \mathrm{ml}, 0,056 \pm 0,002 \mathrm{ml} \mathrm{e} 0,050 \pm 0,003 \mathrm{ml}$. (quadro I)

No grupo das agulhas da marca Ryncos ${ }^{\circledR}$, o volume residual obtido para as agulhas $18,22(30 \times 0,7 \mathrm{~mm}$ e $25 \times 0,7 \mathrm{~mm})$ e $26 \mathrm{G}$ foram respectivamente $0,075 \pm 0,004 \mathrm{ml}, 0,055 \pm 0,004 \mathrm{ml}, 0,056 \pm$ $0,005 \mathrm{ml} \mathrm{e} 0,052 \pm 0,005 \mathrm{ml}$. (quadro II)

A diferença entre os volumes residuais das duas marcas foi estatisticamente significante para as agulhas do tipo $18 \mathrm{G}$, $22 \mathrm{G}(30 \times 0,7 \mathrm{~mm})$ e $26 \mathrm{G}$ com $\mathrm{p}=0,01, \mathrm{p}<0,01$ e $\mathrm{p}<0,01$ respectivamente. Não houve diferença estatisticamente significante do volume residual entre as agulhas do tipo $22 \mathrm{G}(25 \mathrm{x}$ $0,7 \mathrm{~mm})$ das duas marcas $(\mathrm{p}=0,83)$.

\section{DISCUSSÃO}

Diversos oftalmologistas referem o uso de um conjunto de seringa e agulha para aspiração do vítreo, e uma seringa ou duas para a injeção dos antibióticos, mantendo a mesma agu-

\begin{tabular}{|c|c|c|c|}
\hline \multicolumn{4}{|c|}{$\begin{array}{l}\text { Quadro I. Cálculo do volume retido das agulhas da marca Becton-Dickinson }{ }^{\circledR} \text { obtido ao subtrair-se do peso do conjunto completo com } \\
\text { água destilada, o peso da agulha vazia e seca e o peso da seringa apenas com água destilada }\end{array}$} \\
\hline \multirow[b]{3}{*}{ Tipo } & \multicolumn{3}{|c|}{ Agulhas Becton-Dickinson ${ }^{\circledR}$ - peso em gramas } \\
\hline & Agulha seca & Agulha+seringa+água & VR na agulha \\
\hline & Média e DP & Média e DP & Média e DP \\
\hline $18 \mathrm{G}$ & $0,340 \pm 0,004$ & $3,788 \pm 0,005$ & $0,084 \pm 0,004$ \\
\hline $22 \mathrm{G}(30 \times 0,7 \mathrm{~mm})$ & $0,236 \pm 0,003$ & $3,663 \pm 0,004$ & $0,063 \pm 0,002$ \\
\hline $22 \mathrm{G}(25 \times 0,7 \mathrm{~mm})$ & $0,210 \pm 0,002$ & $3,630 \pm 0,003$ & $0,056 \pm 0,002$ \\
\hline $26 \mathrm{G}$ & $0,176 \pm 0,005$ & $3,590 \pm 0,006$ & $0,050 \pm 0,003$ \\
\hline
\end{tabular}




\begin{tabular}{|c|c|c|c|}
\hline \multicolumn{4}{|c|}{$\begin{array}{l}\text { Quadro II. Cálculo do volume retido das agulhas da marca Ryncos }{ }^{\circledast} \text { obtido ao subtrair-se do peso do conjunto completo com água } \\
\text { destilada, o peso da agulha vazia e seca e o peso da seringa apenas com água destilada. }\end{array}$} \\
\hline \multirow[b]{2}{*}{ Tipo } & \multicolumn{3}{|c|}{ Agulhas Ryncos ${ }^{\circledR}$ - peso em gramas } \\
\hline & $\begin{array}{l}\text { Agulha seca } \\
\text { Média e DP }\end{array}$ & $\begin{array}{l}\text { Agulha+seringa+água } \\
\text { Média e DP }\end{array}$ & $\begin{array}{l}\text { VR na agulha } \\
\text { Média e DP }\end{array}$ \\
\hline $18 \mathrm{G}$ & $0,391 \pm 0,003$ & $3,831 \pm 0,005$ & $0,075 \pm 0,004$ \\
\hline $22 \mathrm{G}(30 \times 0,7 \mathrm{~mm})$ & $0,228 \pm 0,002$ & $3,648 \pm 0,005$ & $0,055 \pm 0,004$ \\
\hline $22 \mathrm{G}(25 \times 0,7 \mathrm{~mm})$ & $0,229 \pm 0,003$ & $3,649 \pm 0,005$ & $0,056 \pm 0,005$ \\
\hline $26 \mathrm{G}$ & $0,181 \pm 0,002$ & $3,597 \pm 0,006$ & $0,052 \pm 0,005$ \\
\hline
\end{tabular}

lha. Tal procedimento influencia de forma significativa o volume final injetado, principalmente quando a dose do medicamento está concentrada em um pequeno volume de líquido, podendo nesses casos o compartimento de reserva passar a equivaler a até mais da metade do volume final desejado. Tomemos como exemplo 1, uma injeção de amicacina e vancomicina pós punção vítrea. Na situação atual, o cirurgião deverá realizar a punção com uma seringa de $1 \mathrm{ml}$, e uma agulha de $22 \mathrm{G}$, retirando aproximadamente $0,3 \mathrm{ml}$ de humor vítreo. A partir disso haverá troca apenas de seringa e injeção de $0,1 \mathrm{ml}$ de antibiótico. Sabemos pelo estudo que existe retenção de humor vítreo no compartimento de reserva da agulha de aproximadamente $0,06 \mathrm{ml}$, havendo, portanto, a simples troca da seringa e injeção de $0,1 \mathrm{ml}$ de antibiótico, resultando numa injeção efetiva de apenas $0,04 \mathrm{ml}(40 \%)$ do antibiótico e provável diminuição da eficácia terapêutica.

Fujii et al. alertam para a possibilidade de serem cometidos erros durante a diluição dos antibióticos, baseando-se no fato da existência do compartimento de reserva na ponta da seringa, capaz de suportar um volume de até $0,1 \mathrm{ml}{ }^{(16-17)}$. Ao posicionar o êmbolo na marca de $0,1 \mathrm{ml}$, na realidade teremos dentro da seringa aproximadamente $0,2 \mathrm{ml}$, o que pode resultar na obtenção do dobro de concentração considerada segura dos antibióticos de administração intravítrea e implicar em danos adicionais a uma retina já em sofrimento pela infecção ${ }^{(3)}$.

Assim como o compartimento de reserva contido no bico da seringa tem que ser valorizado pelo oftalmologista na diluição das soluções de uso intravítreo, este também deve ser valorizado no momento da injeção do medicamento intravítreo. Tal raciocínio completo pode ser ilustrado pelo seguinte exemplo 2 : sabemos que a maioria dos tratamentos para endoftalmite incluem pelo menos dois antibióticos intravítreos. Os resultados do estudo atual mostram que a agulha de insulina (26 G), após desacoplada da seringa no final de uma injeção, ainda retém um volume de aproximadamente $0,05 \mathrm{ml}$. Isso significa que, se após a injeção de $0,1 \mathrm{ml}$ do primeiro antibiótico, na tentativa de evitar uma segunda punção, for aproveitada a mesma agulha para injeção do segundo antibiótico, podemos pressupor que estamos injetando um volume de $0,05 \mathrm{ml}$ do primeiro antibiótico retido e mais $0,05 \mathrm{ml}$ do segundo, pois os $0,05 \mathrm{ml}$ restantes ficarão retidos no compartimento de reserva da agulha. Como resultado final teremos aproximadamente a aplicação de $0,15 \mathrm{ml}$ do primeiro antibiótico e $0,05 \mathrm{ml}$ do segundo, diferente do plane- jado de $0,1 \mathrm{ml}$ de cada antibiótico, alterando a proporção entre os antibióticos de $1: 1$ para $3: 1^{(12,16-17)}$.

Os erros são ainda maiores quando agulhas de maior calibre (18 G e $22 \mathrm{G})$ são utilizadas na aspiração do vítreo e injeção do antibiótico, pois irá resultar na reintrodução do vítreo do compartimento de reserva para a cavidade vítrea e injeção de subdoses dos antibióticos, como demonstramos no exemplo1.

Lembrar sempre que previamente à injeção dos antibióticos, deve-se empurrar o êmbolo da seringa até a marcação do volume desejado, já com a agulha acoplada à seringa, de modo que o lume da agulha também esteja preenchida com antibiótico.

Os métodos de injeção intravítreo aparentemente adequados são os seguintes (figura 1):

1 - Aspiração do vítreo por um conjunto de seringa e agulha que será desprezada e aplicação dos antibióticos misturados em um outro conjunto de agulha e seringa, num total de 2 punções.

2 - Aspiração do vítreo por um conjunto de seringa e agulha que será desprezada e, aproveitando uma mesma agulha para os dois antibióticos, fazendo apenas a troca das seringas e conhecendo o volume do compartimento de re serva da agulha, aplicar um volume menor do primeiro an tibiótico e maior do segundo antibiótico, num total de 2 punções.

3 - Aspiração do vítreo por um conjunto de seringa e agulha que será desprezada e aplicação dos antibiótico por dois conjuntos de seringas e agulhas contendo em cada um deles um antibiótico, num total de 3 punções.

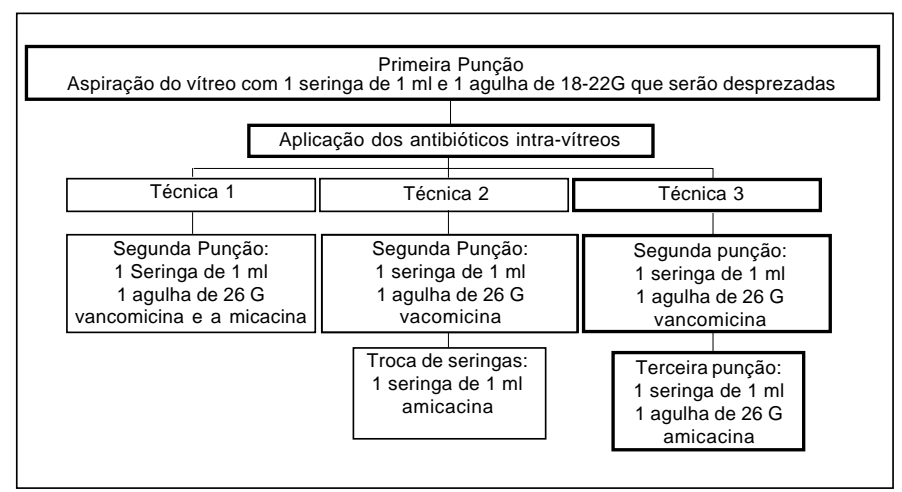

Figura 1 - Fluxograma dos métodos de injeção intravítrea 
Sabemos que vancomicina e ceftazidima são fisicamente incompatíveis, portanto devem ser injetados obrigatoriamente em seringas separadas ${ }^{(1)}$. Apesar de não existir relatos de incompatibilidade físico-química entre vancomicina e amicacina ${ }^{(18)}$, como princípio de terapêutica antibiótica, deve-se evitar a mistura dos antibióticos numa mesma solução, pois tal procedimento pode provocar interação farmacocinética das drogas tornando a sua eficácia imprevisíveis, inviabilizando assim a técnica 1.

A aplicação dos antibióticos com a mesma agulha, mesmo conhecendo o volume do compartimento de reserva da agulha, não é uma técnica confiável, pois como foi mostrado no estudo atual existe variação do volume do compartimento de reserva entre as diferentes marcas de agulhas, tornando assim a técnica 2 pouco confiável.

Diante destas observações podemos concluir que a terceira técnica (em negrito no fluxograma), ou seja, aspiração do vítreo por um conjunto de seringa e agulha que será desprezada e aplicação dos antibiótico por dois conjuntos de seringas e agulhas contendo em cada um deles um antibiótico, num total de 3 punções, ainda é a melhor técnica de aplicação de antibiótico intravítreo, alcançando um volume injetado mais próximo do planejado.

\section{ABSTRACT}

Purpose: To assess evaluate the accuracy of intravitreal drug administration in the treatment of endophthalmitis and to suggest a reproducible and accessible technique for this procedure. Methods: To assess the retained volumes in needles used for the intravitreal injection of antibiotics using an analytical scale. Results: Means and standard deviations of retained volumes in the $26,22(25 \times 0.7 \mathrm{~mm}$ and $30 \times 0.7 \mathrm{~mm})$ and $18 \mathrm{G}$ were respectively $0.051 \pm 0.006,0.056 \pm 0.005,0.055 \pm 0.004$ and $0.075 \pm 0.004$ for needles Ryncos ${ }^{\circledR}$ and $0.050 \pm 0.003,0.056 \pm 0.002,0.063 \pm 0.002$ and $0.084 \pm 0.004$ for Becton-Dickinson ${ }^{\circledR}$. There were statistically significant differences in the retained volumes between the two needle brands for $26,22(30 \times 0.7 \mathrm{~mm})$ and $18 \mathrm{G}$ needles with $\mathrm{p}=$ $0.01, p<0.01$ and $p<0.01$ respectively. No difference was found only for $25 \times 0.7 \mathrm{~mm} 22 \mathrm{G}$ needle with $\mathrm{p}=0.83$. Conclusion: Most needles used for intravitreal injection hold a retained volume in the reserve needle compartment that should be considered during the injection technique, therefore minimizing errors during intravitreal injection of antibiotics.
Keywords: Endophthalmitis/drug therapy; Vitreous body/drug effects; Antibiotics/administration \& dosage; Injections/ methos; Medication errors

\section{REFERÊNCIAS}

1. Kresloff MS, Castellarin AA, Zarbin MA. Endophthalmitis. Surv Ophthalmol 1998;43:193-224

2. Baum J, Peyman GA, Barza M. Intravitreal administration of antibiotic in the treatment of bacterial endophthalmitis. Surv Ophthalmol 1982;26:204-6.

3. Fujii GY, Moreira Jr CA, Scapucin L, Fujii LJ. Retinal toxicity to antibiotics: an alert to the correct dilution procedure of intravitreal antibiotics. Rev Bras Oftal. 1999;58:669-72.

4. Roth DB, Flynn HW. Antibiotic selection in the treatment of endophthalmitis: the significance of drug combinations and synergy. Surv Ophthalmol 1997;41:395-401

5. Peyman GA. Antibiotic administration in the treatment of bacterial endophthalmitis. II. Intravitreal injections. Surv Ophthalmol 1977;21:332, 339-46.

6. Pflugfelder SC, Hernández E, Fliesler SJ, Alvarez J, Pflugfelder ME, Forster RK. Intravitreal vancomycin. Retinal toxicity, clearence, and interaction with gentamicin. Arch Ophthalmol 1987;105:831-7.

7. Campochiaro PA. Lim JI. Aminoglycoside toxicity in the treatment of endophthalmitis. The Aminoglycoside Toxicity Study Group. Arch Ophthalmol 1994; 112:48-53.

8. Homer P, Peyman GA, Koziol J, Sanders D. Intravitreal injection of vancomycin in experimental staphylococcal endophthalmitis. Acta Ophthalmol 1975;53:311-20.

9. Oum BS, D'Amico DJ, Wong KW. Intravitreal antibiotic therapy with vancomycin and aminoglycoside. An experimental study of combination and repetitive injections. Arch Ophthalmol 1989;107:1055-60.

10. Aaberg TM, Flynn HW, Murray TG. Intraocular ceftazidime as an alternative to the aminoglycosides in the treatment of endophthalmitis. Arch Ophthalmol 1994;112:18-9.

11. Noske, W. Inaccuracy in preparation of intravitreal solutions. Arch Ophthalmol 1986;104:1748-9.

12. Turco SJ. Devices. In: Turco SJ. Sterile Dosage Forms: their preparation and clinical application. Philadelphia: Lea \& Febiger; 1994. p.324-31.

13. Olk RJ, Bohigian GM. The management of endophthalmitis: diagnostic and therapeutic guidelines including the use of vitrectomy. Ophthalmic Surg 1987; 18:262-7.

14. Donahue SP, Kowalski RP, Eller AW, DeVaro JM, Jewart BH. Empiric treatment of endophthalmitis. Are aminoglycosides necessary? Arch Ophthalmol 1994;112:45-7.

15. Langston-Pavan D. Uveal tract: iris, ciliary body, and choroid. In: LangstonPavan D. Manual of ocular diagnosis and therapy. United States of America: Little, Brown; 1996. p.218-9.

16. Associação Brasileira de Normas Técnicas. Seringas de plástico estéreis e de uso único para uso manual-especificação (NBR9752). Rio de Janeiro: ABNT;1997. p.1-14.

17. International Standard Organization. Sterile hypodermic syringes for single use (ISO 7886). Geneva: ISO;1984. p1-13.

18. Reynolds JEF. Antibacterial Agents. In: Reynolds JEF. Martindale: The extra pharmacopoeia. London: The Royal Pharmaceutical Society;1996. p.295-6.

Nos artigos enviados para publicação, o nome dos autores

e suas afiliações devem estar completos. Isso facilitará a indexação e os links com as bases de dados e o CV Lates. 